\title{
Research on Block-Based Blind Identification of High-rate Punctured Convolutional Codes
}

\author{
Hua Yan ${ }^{1, *}$, Ying Huang ${ }^{1}$, Baoguo $\mathrm{Li}^{1}$, and Jing Lei ${ }^{1}$ \\ ${ }^{1}$ College of Electronic Science, National University of Defense Technology, Chang Sha, Hunan Province, China
}

\begin{abstract}
Aiming at the large amount of memory in high bit rate punctured convolutional codes, a soft recognition algorithm based on block Hadamard matrix (B-SWHT) is proposed. By using the idea of block Hadamard matrix operation, the soft WHT algorithm with excellent fault-tolerance performance is applied to the recognition of high-rate punctured convolutional codes. The simulation experiments show that the algorithm can effectively reduce the memory required for the recognition of high-rate punctured convolutional codes while maintaining high fault-tolerance performance. This paper provides a feasible solution for the identification of high bit rate punctured convolutional codes.
\end{abstract}

\section{Introduction}

In the face of poor and time-varying wireless communication channels, in order to ensure communication quality, a communication system is usually designed based on the worst-case channel conditions. However, this also causes a problem that the channel capacity cannot be fully utilized. Adaptive Modulation and Coding (AMC) can adjust the parameters of the transmitter (such as modulation method and coding method) in real time according to the change of channel quality, so as to ensure that the channel is fully utilized. Because the channel environment is constantly changing, the parameter information of the transmitter is limited by various aspects in practical applications and cannot be transmitted to the receiver in real time. Therefore, how to quickly identify the channel coding system based on the signal and data received by the receiver is the key to recovering the original information sequence. This shows that the analysis and identification of channel coding play an important role in practical communication applications.

Because of its simple structure, variable code rate, and strong versatility of decoding, punctured convolutional codes have been widely used in satellite communication systems, mobile communication systems, and deep space communication systems, and their identification methods have also become a hot topic. Literature [1] proposed a method of identifying punctured convolutional codes by using the constraint relationship between generator polynomial matrix and check polynomial matrix. The disadvantage of this method is that there are many unknowns in the process of solving the linear equations, and the number of iterations of the algorithm is larger, which leads to higher recognition complexity. A $1 / n$ code rate convolutional code is divided into a number of $1 / 2$ code rate convolutional codes, and Walsh-Hadamard transform is used to solve the $1 / 2$ code rate convolutional code check matrix [2]. This realizes the blind recognition of a $1 / n$ convolutional code at a high error rate. However, this method is limited by the memory space of the computer and cannot solve the high-dimensional check matrix. According to the soft information of the received sequence, the cumulative log-likelihood ratio of the code-word verification relationship is calculated, and the recognition accuracy is estimated [3]. This method fully utilizes the reliability information of the bit-symbols to improve the faulttolerant capability of the code recognition. However, the problem with this method is that the soft decision algorithm is based on a specific set of code constraints to identify it, and it cannot perform full blind recognition. For the problem of hard-decision loss of a large amount of sequence information, it is proposed to introduce the sequence soft information into the frequency sequence to improve the fault-tolerant performance of the algorithm [4]. Due to the limitation of the memory space of the computer, using the WHT algorithm does not identify the punctured convolutional code with a higher code rate. In summary, the WHT algorithm is widely used. However, the algorithm requires a large amount of storage when it is used to identify high-rate punctured convolutional codes, which exceeds the range that the computer can handle. In this paper, based on the WHT algorithm of soft decision sequences, this paper proposes a blind recognition algorithm based on block Hadamard matrix for high bit rate punctured convolutional codes. While taking full advantage of the fault-tolerant performance of the WHT algorithm, the algorithm can effectively reduce the computational memory required for identifying high-rate punctured convolutional codes

\footnotetext{
* Corresponding author: 28113901@qq.com
} 
through block-based Hadamard matrix operations. Therefore, it has good application value.

\section{Punctured convolutional code}

Punctured convolutional code can generally construct a punctured convolutional code of any $n-1 / n$ code rate by a convolutional code with a code rate of $1 / 2$ [5].Therefore, in this paper, we focus on various optimal punctured convolutional codes generated by $1 / 2$ code rate convolutional codes, and illustrate the blind recognition algorithm based on block Hadamard matrix.

Let $\mathrm{F}$ be a binary domain, $F[D]$ be a polynomial ring, and $F(D)$ be a rational function domain. A convolutional code with a code rate of $1 / 2$ can be represented as any one-dimensional subspace $\mathrm{C}$ on $F(D)^{2}$. If $G(D)$ is a matrix of $1 \times 2$ on $F(D)$ and the row vector forms a set of bases of C, $G(D)$ is said to be A generator matrix for C. If $G(D)$ is a polynomial generator matrix and $G(0)$ is full rank, $G(D)$ is called an encoding matrix.

For a convolutional code $\mathrm{C}$ with a code rate of $1 / 2$, the information sequence $U(D)=\sum_{i=0}^{\infty} u_{i} D^{i}$ is input, and the coded output code sequence is $V(D)=\left(\sum_{i=0}^{\infty} v_{i, 0} D^{i}, \sum_{i=0}^{\infty} v_{i, 1} D^{i}\right)$. By constructing a highrate punctured code by the $1 / 2$-rate convolutional code, it is first necessary to equivalently split the information sequence and the code sequence, and then delete the specific position of the output codeword. Finally, the code with the increased code rate is obtained. As shown in Figure 1.

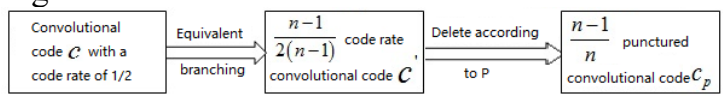

Fig. 1. Construction of a punctured convolutional code

\section{Recognition of punctured convolutional codes based on B-SWHT}

\subsection{Walsh-Hadamard algorithm}

According to the theorem of punctured convolutional codes, a punctured convolutional code with an arbitrary code rate $n-1 / n$ has only a unique minimum check polynomial. Therefore, the classical Walsh-Hadamard transform algorithm can identify the simplest check polynomial of the punctured convolutional code of the equivalent code rate by using the conventional $n-1 / n$ convolutional code generating codeword. The recognition principle of WHT algorithm [2] is as follows.

Let $H_{P}(D)^{T}=\left(h_{0}(D), h_{1}(D), \cdots, h_{n-1}(D)\right)$ be the check polynomial matrix of $C_{P}$, and use the relationship between the convolutional code generation matrix and the check matrix $G_{P}(D) \cdot H_{P}(D)^{T}=0$, when the received data length $N>n \times(m+1)$ (where $\mathrm{m}$ is the coding constraint degree).According to the received sequence $R_{P}(D)$ in the case of high bit error, a parity check matrix of $C_{P}$ can be obtained by solving a set of solution vectors satisfying the largest number of equations in the system of the faulty linear equations in equation (1).

$$
\sum_{j=0}^{m} \sum_{i=1}^{n} r_{i, j+s} h_{i, \mathrm{~m}-j}=0, s=0,1, \cdots, N
$$

Convert (1) to a system of binary equations:

$$
\left[\begin{array}{ccccccc}
r_{1,0} & \cdots & r_{n, 0} & \cdots & r_{1, \mathrm{~m}} & \cdots & r_{n, \mathrm{~m}} \\
r_{1,1} & \cdots & r_{n, 1} & \cdots & r_{1, \mathrm{~m}+1} & \cdots & r_{n, \mathrm{~m}+1} \\
\vdots & & \vdots & & \vdots & & \vdots \\
r_{1, N} & \cdots & r_{n, N} & \cdots & r_{1, \mathrm{~m}+N} & \cdots & r_{\mathrm{n}, \mathrm{m}+N}
\end{array}\right]\left[\begin{array}{c}
h_{1, \mathrm{~m}} \\
\vdots \\
h_{n, \mathrm{~m}} \\
\vdots \\
h_{1,0} \\
\vdots \\
h_{n, 0}
\end{array}\right]=0
$$

The equations (2) indicate that the received sequence $R_{P}(D)$ is grouped by lengths, each set of codeword vectors is expressed as a decimal number, and the resulting frequency sequence $\eta=\left[\eta_{0}, \eta_{1}, \cdots, \eta_{2^{n(m+1)}-1}\right]$ is constructed using the obtained decimal numbers. Then perform WHT operation on $\eta$ :

$$
z=\eta \cdot H_{2^{n(m+1)}}
$$

In the formula, $z=\left[z_{0}, z_{1}, \cdots, z_{2^{n(m+1)}-1}\right]$ is a Walsh spectrum of $\eta$, and $H_{2^{n(m+1)}}$ is a Hadamard matrix of order $n(m+1)$. The binary vector $i_{\text {bin }}$ corresponding to the position of the largest element $z_{i}$ in the Walsh spectrum is the check sequence of the encoding.

\subsection{Soft decision frequency sequence}

The classical Walsh-Hadamard transform method is based on the hard-decision 0,1 sequence of the received sequence to construct the frequency coefficient, because the decision function has only 0,1 values, and the frequency coefficient can not reflect the size of the judgment probability, thus resulting in the loss of estimation performance. The soft decision frequency sequence is used in [3] to make full use of the reliability information of bit symbols to improve the fault tolerance of code recognition.

The model of the communication system is shown in Figure2. The information bit $b=\left[b_{1}, b_{2}, \cdots, b_{k}\right]$ is coded to generate a code sequence $c=\left[c_{1}, c_{2}, \cdots, c_{n}\right]$, and after adding a specific modulation method, it is transmitted through the channel. The received symbol can be represented as $r_{i}=s_{i}+n_{i}$, where $s_{i}$ represents the BPSK 
modulation symbol (binary $0 \rightarrow+1,1 \rightarrow-1$ ) and $n_{i}$ represents additive white Gaussian noise (mean value 0 , variance is $\left.N_{0} / 2\right)$. Using the received sequence $r$, the soft information $\ell=\left[\ell_{1}, \cdots, \ell_{n}\right]$ of the codeword $C$ can be calculated, where $\ell_{i}$ represents the posterior log likelihood ratio (LLR) of the codeword $c_{i}$ :

$$
\ell_{i}=\log \left(\frac{\operatorname{Pr}\left(c_{i}=0 \mid r\right)}{\operatorname{Pr}\left(c_{i}=1 \mid r\right)}\right)
$$

Assuming that the BPSK signal is transmitted over the AWGN channel, the soft information can be expressed as:

$$
\ell_{i}=\Lambda\left(c_{i} \mid r_{i}\right)=\frac{4 r_{i}}{N_{0}}
$$

At the same time, the received sequence $r$ is grouped according to the length $n(m+1)$ and is composed of $N$ decision equation coefficient vectors $r_{0}, r_{1}, \cdots, r_{N-1}$. We can define the sequence vector soft decision function as:

$$
f_{\text {soft }}\left(\boldsymbol{r}_{i}, k\right)=\left\{\begin{array}{cc}
\underset{j=0, \cdots, n(m+1)-1}{\boxplus} \ell_{j}^{i}, & , r_{i, d e c}=k \\
0 & , r_{i, d e c} \neq k
\end{array}\right.
$$

among them

$$
\ell_{1} \boxplus \ell_{2}=\operatorname{sign}\left(\ell_{1}\right) \operatorname{sign}\left(\ell_{2}\right) \min \left(\left|\ell_{1}\right|\left|\ell_{2}\right|\right)+f\left(\mid \ell_{1}+\right.
$$

The soft-decision frequency coefficients available for sequence vectors are:

$$
\eta_{k}=\sum_{i=0}^{N-1} f_{\text {soft }}\left(r_{i}, k\right), k=0,1, \cdots, 2^{n(m+1)}-1
$$

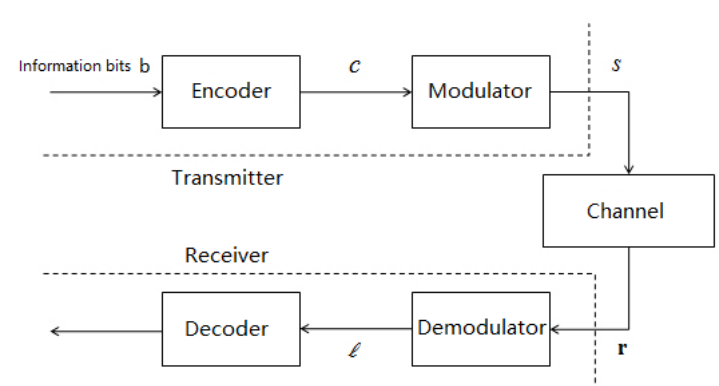

Fig. 2. Communication system model

\subsection{Identify check polynomials based on block Hadamard matrix}

The punctured convolutional code improves the code rate and the transmission rate, but its high dimensionality limits the application of the traditional WHT algorithm. For a $n-1 / n$ punctured convolutional code generated by a $1 / 2$ source convolutional code. In the calculation of the traditional WHT algorithm, the Hadamard matrix has a size of $2^{n(m+1)} \times 2^{n(m+1)}$ and requires $4^{n(m+1)}$ memory to store it. When the code rate of the convolutional code is removed by $7 / 8$ and the code memory $\mathrm{m}=6$, the required memory reaches the order of $10^{34}$ bit, which far exceeds the storable space of the computer. In this regard, this paper proposes an algorithm based on the block Hadamard matrix, which greatly reduces the required operating storage space, enabling the WHT algorithm to handle the identification of high-dimensional punctured convolutional codes. The algorithm identification process is as follows:

Step 1: Assume that the received sequence of the $(n, n-1, m)$ punctured convolutional code is $R_{P}(D)$, group $R_{P}(D)$ into $n(m+1)$ lengths, and represent each set of codeword vectors as a decimal number. The resulting $N$ decimal numbers are used to construct the frequency sequence $\eta=\left[\eta_{0}, \eta_{1}, \cdots, \eta_{2^{n(m+1)}-1}\right]$.

Step 2: When $n(m+1)$ is an even number, divide the $n(m+1)$-length vector of codewords into equal parts, that is, divide $\eta$ by $2^{n(m+1) / 2}$ to get $\eta_{d}=\left[\eta_{d_{0}}, \eta_{d_{1}}, \cdots, \eta_{d_{2^{(m+n+1) / 2-1}}}\right]$.

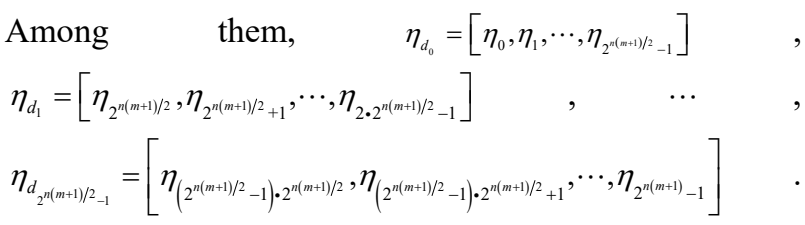
When $n(m+1)$ is odd, the difference is only that the separation length is different.

Step 3: According to the frequency sequence after the equalization, the $2^{n(m+1)}$-order Hadamard matrix is correspondingly divided into $2^{n(m+1) / 2} \quad 2^{n(m+1) / 2}$-order Hadamard matrices, namely:

$$
H_{2^{n(m+1)}}=\left[\begin{array}{cccc}
H_{2^{n(m+1) / 2}} & H_{2^{n(m+1) / 2}} & \cdots & H_{2^{n(m+1) / 2}} \\
H_{2^{n(m+1) / 2}} & H_{2^{(m+1) / 2}} & \cdots & H_{2^{n^{(m+1) / 2}}} \\
\vdots & \vdots & & \vdots \\
H_{2^{n(m+1) / 2}} & H_{2^{n(m+1) / 2}} & \cdots & H_{2^{n^{(m+1) / 2}}}
\end{array}\right]
$$

Step 4: Calculate the equidistant frequency sequence and the block Hadamard matrix to obtain the spectral coefficients of the block algorithm:

$$
z_{d}{ }^{T}=\left[z_{d_{0}}, \mathrm{z}_{d_{1}}, \cdots, z_{d_{2^{n(m+1) / 2-1}}}\right]^{T}=H_{2^{n(m+1)}}{ }^{T} \cdot \eta_{d}^{T}
$$

Step 5: Determine the position of the largest element in the entire Walsh spectral coefficient by comparing the largest elements in the $2^{n(m+1) / 2} z_{d_{i}}$, so that the check sequence can be obtained.

From the above algorithm identification process, it can be seen that once a block Hadamard algorithm 
reduces the Hadamard matrix operation that originally requires $2^{n(m+1)} \cdot 2^{n(m+1)}=2^{2 n(m+1)} b i$ storage space to only $2^{n(m+1) / 2} \cdot 2^{n(m+1) / 2}=2^{n(m+1)} b i t$, the algorithm can continue to perform secondary blocking and reduce the required storage space to $2^{n(m+1) / 2}$ bit size. It can be seen that there is a significant reduction in operating storage requirements compared to the classic WHT algorithm.

\section{Simulation and analysis}

Consider the $1 / 2$ code rate convolutional codes of four different constraint degrees, ie, the $(2,1,2),(2,1,3) 、(2,1,4) 、(2,1,5)$ convolutional codes, and perform the WHT algorithm operations on the soft and hard decision frequency sequences respectively. Through Monte Carlo experiments, the recognition performance of the two recognition algorithms for different convolutional codes is compared. The simulation results are shown in Figure 3.

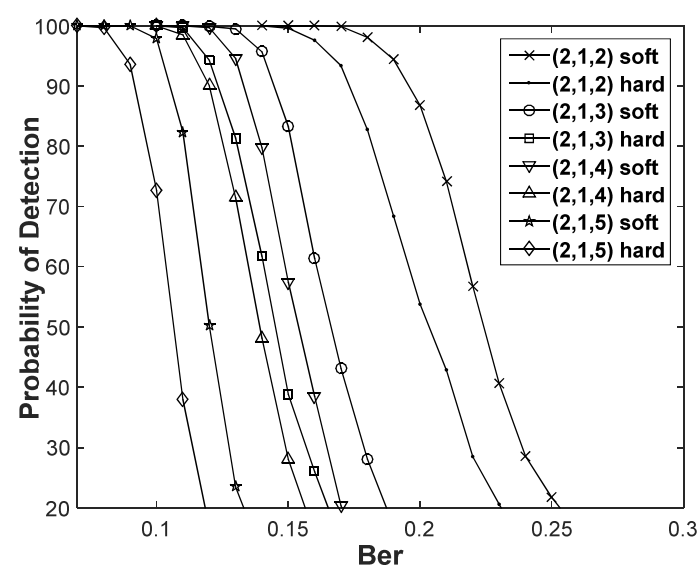

Fig. 3.WHT algorithm performance comparison of soft and hard-decision frequency sequences with $1 / 2$ code rate convolutional code

Among them, the number of simulations is 1000 , and the number of decision equations is $N=1000$. Using BPSK modulation, the channel model is AWGN model. It can be seen from the experimental results that the performance of the WHT algorithm is significantly improved by the soft decision frequency sequence.For example, when the $(2,1,2)$ convolutional code is at a bit error rate of 0.2 , the hard-decision-frequency sequence WHT algorithm can only achieve $50 \%$ correct recognition probability, and the soft-decision frequency sequence WHT algorithm can achieve more than $85 \%$ correct recognition probability.

The identification of punctured convolutional codes with $n-1 / n$ code rate is based on a $1 / 2$ code rate source convolutional code. On the $1 / 2$ code rate convolutional codes that the classic WHT algorithm can handle, the block Hadamard matrix algorithm is used to reduce the running memory of the algorithm while not reducing the recognition performance. For the different code rate punctured convolutional codes generated on the basis of the $(2,1,6)$ convolutional code, when using the block Hadamard algorithm, the reduction of the running storage volume is shown in Figure 4 .As we can be seen from the figure, the algorithm based on the block Hadamard matrix reduces the amount of running memory required to identify high-rate punctured convolutional codes to the computer-processable range $\left(10^{9}\right)$. For example, for a $7 / 8$ code rate convolutional code generated on the basis of a $(2,1,6)$ convolutional code, the running memory amount is reduced from $10^{33}$ bit to $10^{8}$ bit after passing twice a block Hadamard algorithm.

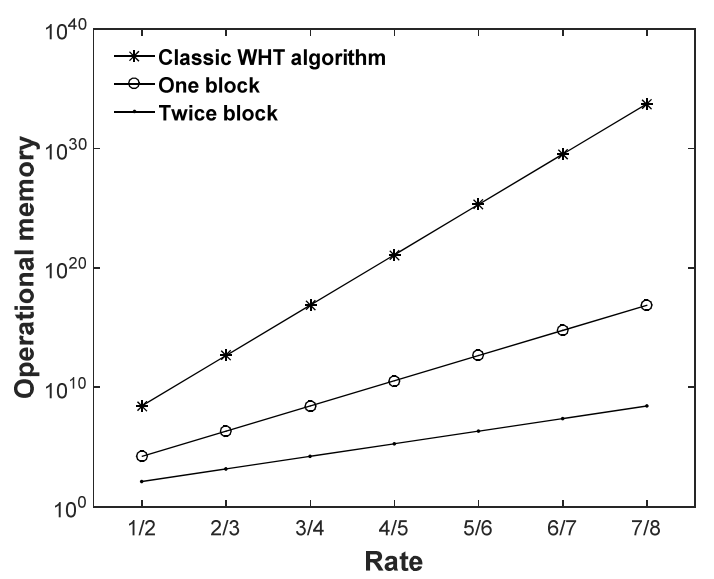

Fig. 4. Operational storage of different code rate punctured convolutional codes

The soft decision sequence algorithm based on block Hadamard matrix proposed in this paper identifies the syndrome relations of punctured convolutional codes. Figure 5 shows the recognition performance of the $2 / 3$ code rate convolutional code generated by the $(2,1,6)$ convolutional code when the received data amount is 21000. It can be seen from the figure that the soft decision recognition algorithm based on the block Hadamard matrix can not only reduce the running storage to the computer-processable range, but also maintain the recognition performance of the classic WHT algorithm at high bit error rate.

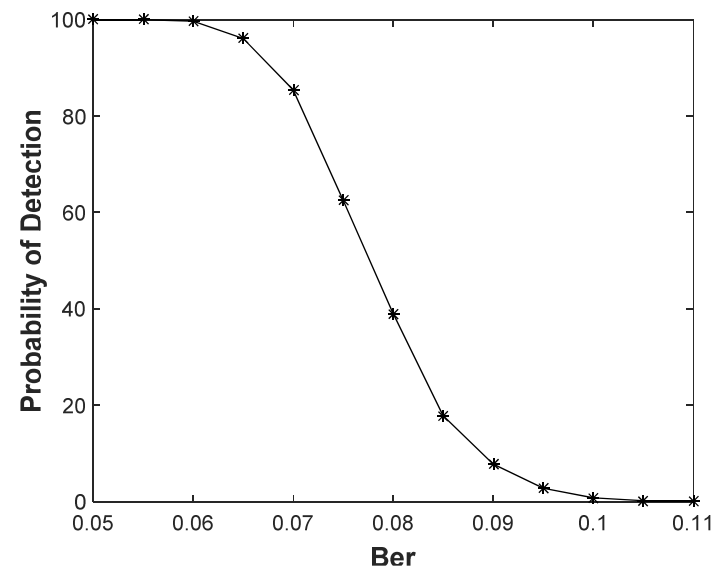

Fig. 5.The B-SWHT algorithm recognition performance of $2 / 3$ code rate punctured convolutional codes generated with $(2,1,6)$ convolutional codes 


\section{Conclusions}

This paper proposes a punctured convolutional code recognition algorithm based on B-SWHT and compares the fault-tolerance performance of $1 / 2$ code rate convolutional codes with different constraint degrees under the WHT algorithm of soft and hard decision sequence, this verifies that the soft decision reliability information improves the fault-tolerant performance of the WHT algorithm. By introducing soft decision frequency sequences, the combination of soft information and the classic WHT algorithm is realized, which ensures the excellent fault tolerance of the algorithm. At the same time, the idea of block Hadamard matrix is used to solve the problem of high storage capacity for high-rate deconvolution. Based on the code rate punctured convolutional codes generated on the basis of $(2,1,6)$ convolutional codes, the reduction of the running storage capacity after using the block Hadamard algorithm is analyzed. The simulation performance of the $2 / 3$ code punctured convolutional code generated by the $(2,1,6)$ convolutional code is analyzed. It can be seen from the simulation results that using this algorithm, excellent fault tolerance can be guaranteed. And can reduce the amount of running storage to a range that the computer can handle. Therefore, this algorithm has important application value for the recognition of high bit rate punctured convolutional codes. With the increase of code rate, the time complexity of the classic WHT algorithm is an area that needs further research and improvement.

\section{References}

1. Lu Pei-zhong, Shen Li, Zou Yan, etal. Blind recognition of punctured convolutional codes[J].Science in china Ser Information Sciences,2005,48(4):484-498.

2. Liu Jian, Wang Xiao-jun, Zhou Xi-yuan, Blind Recognition of Convolutional Coding Based on Walsh-Hadamard Transform[J],Journal of Electronic and Information Technology, 2010,32(4):884-888.

3. R. Moosavi and E. G. Larsson ,"Fast blind recognition of channel codes," IEEE Trans. Commun., vol.62,no.5,pp.1393-1405,May 2014.

4. HUANG Kai-zhi, CHEN Song. A soft fast estimation method of PN sequence based on weighting Walsh- Hadamard transform (WHT) [J]. Journal of Electronic \& Information Technology , $2013,35(1)$ : 74-79.

5. SHEN B Z, PATAPOUTIAN A, McEWEN P A. Punctured recursive convolutional encodes and their applications in turbo codes[J]. IEEE Trans on inform Theory,2001,47(5):2300-2320.

6. You Ling, Zhu zhong-liang, The application of Walsh Function in Resolving of $F(2)$ equations[J].Signal Processing, 2000,16 (12) : 27-31.
7. J. G. Proakis and M. Salehi, Digital Communication, 5th ed. McGraw-Hill,2008.

8. Qi Lin, Hao Shi-qi, Wang Lei, Blind decoding algorithm of punctured convolutional codes based on improved WHT[J], Application Research of Computers, 2011,28 (4) : 1457-1459.

9. Yu Pei-dong, Li Jing, Peng Hua, A Novel Algorithm for Channel Coding Recogniton Using SoftDecision[J], Acta Electronica Sinica,2013, 41 (2) : 301-306.

10. Peidong $\mathrm{Yu}$, Hua Peng, Jing Li,“On Blind Recognition of Channel Codes Within a Candidate Set,'IEEE Commun.Lett.,vol.20,no.4,pp.736739,Apr.2016. 